\title{
PERSPECTIVAS DO NOVO SISTEMA DE AVALIAÇÃO DA PÓS-GRADUAÇÃO E OS DESAFIOS DA ÁREA DO DIREITO
}

\author{
Gianpaolo Poggio Smanio ${ }^{1}$ \\ Tais Ramos $^{2}$
}

\section{Introdução}

O ambiente da pós-graduação deve ser aquele em que se estimule o avanço e o desbravamento das fronteiras do conhecimento, sem imposição de barreiras e com atenção à sociedade, promovendo o diálogo entre pares em nível nacional e internacional. O objetivo da pós-graduação brasileira nas últimas décadas foi fundamentalmente a formação de docentes e pesquisadores para o sistema acadêmico nacional. A nova perspectiva é ampliar sua atuação na formação de pessoal qualificado para enfrentar novos desafios científicos com independência intelectual, contribuindo para o desenvolvimento científico, tecnológico, social e econômico do Brasil.

A verificação dessa qualidade se dá por meio de processo de avaliação do sistema nacional de pós-graduação da Coordenação de Aperfeiçoamento de Pessoal de Nível Superior (Capes), o qual requer aperfeiçoamentos conceituais e operacionais, pois transformações significativas nos cenários nacionais e internacionais requerem novas ações das comunidades acadêmica, científica, tecnológica e de inovação, sinalizando para a necessidade de atualização dos procedimentos e dos critérios do modelo de avaliação.

Demandas de várias ordens emergem, como aquelas relacionadas à consolidação, à internacionalização, à inovação e à interação estruturada do Sistema Nacional de Pós-graduação (SNPG) com setores extra-acadêmicos, em especial aqueles diretamente conectados com o processo de transferência de conhecimento para a

\footnotetext{
1 Doutor e mestre em Direito pela Pontifícia Universidade Católica de São Paulo (PUC-SP) e graduado em Direito pela Universidade de São Paulo (USP). É diretor e professor titular da Faculdade de Direito e professor permanente do Programa de Mestrado e Doutorado em Direito Político e Econômico da Universidade Presbiteriana Mackenzie (UPM). Foi coordenador do Programa de Pós-graduação em Direito Político e Econômico da Universidade Presbiteriana Mackenzie (UPM) (2013-2020) e procuradorgeral de justiça do Estado de São Paulo (2016-2018 e 2018-2020). É coordenador adjunto da Comissão Solidariedade e Integração Regional e membro da Comissão APCN da área do Direito da Capes. Procurador de justiça.

2 Doutoranda em Direito Político e Econômico na Universidade Presbiteriana Mackenzie (UPM), com período de doutorado sanduíche no país (SWP) do CNPq na Universidade Federal do Rio Grande do Sul (UFRGS). Graduada em Direito, especialista em Direito Processual Civil e mestre em Direitos Sociais e Políticas Públicas pela Universidade de Santa Cruz do Sul (Unisc). É professora da Faculdade de Direito e membro da Comissão de Planejamento Quadrienal e Autoavaliação do Programa de Pós-graduação em Direito Político e Econômico da Universidade Presbiteriana Mackenzie (UPM). Advogada.
} 
sociedade e maior protagonismo no desenvolvimento socioeconômico, bem como na redução das assimetrias regionais. ${ }^{3}$

Nesse sentido, questiona-se: quais são as perspectivas da nova avaliação baseada em dimensões e quais os desafios dos programas de pós-graduação da área do Direito?

Para responder ao questionamento, analisa-se, num primeiro momento, o atual sistema nacional de avaliação e, num segundo momento, são investigadas as perspectivas do novo modelo de avaliação multidimensional.

Dessa forma, utiliza-se o método dedutivo como abordagem para o desenvolvimento da pesquisa e as técnicas documentais da Capes e, também, fontes legislativas.

2. Sistema Nacional de Pós-graduação (SNPG) e os instrumentos de avaliação

A Coordenação de Aperfeiçoamento de Pessoal de Nível Superior (Capes), fundação do Ministério da Educação (MEC), exerce papel fundamental na expansão e consolidação da pós-graduação stricto sensu (mestrado e doutorado) e na formação de professores da educação básica.

Entre as linhas de atuação da Capes, estão a avaliação da pós-graduação stricto sensu, o acesso à divulgação da produção científica, investimentos na formação de recursos humanos no país e no exterior, a promoção da cooperação científica nacional e internacional e, ainda, a indução e o fomento da formação inicial e continuada de professores para a educação básica nos formatos presencial e a distância.

O Sistema Nacional de Pós-graduação (SNPG) consolidou-se ao longo das últimas décadas ganhando visibilidade nacional e internacional. O sistema está formado atualmente por cursos acadêmicos (3.653 mestrados e 2.405 doutorados) e profissionais (852 mestrados e 37 doutorados) distribuídos por todas as áreas do conhecimento. Esse sistema resulta de esforços conjugados de organizações diversas, com destaque para as universidades, as instituições e os centros de pesquisa e seus respectivos programas de pós-graduação. Idêntico destaque cabe à atuação das agências de fomento CNPq, às Fundações de Amparo à Pesquisa (FAPs) e à centralidade do papel da Capes. ${ }^{4}$

Dentre as ações da Capes, tem especial importância para a qualidade e o sucesso alcançado pela pós-graduação brasileira o processo de avaliação, que orienta a formação pós-graduada stricto sensu de recursos humanos para a produção intelectual

\footnotetext{
BRASIL. Relatório Técnico DAV - Avaliação Multidimensional PPG 2019. Brasília: Ministério da Educação/Capes, 2019

$4 \quad$ Ibidem.
} 
de impacto científico dos grupos de pesquisa, para o alto desempenho de processos econômicos inovadores e para a necessária e obrigatória ampliação da qualidade do trabalho acadêmico e elevação da dinâmica produtiva do país. A avaliação contribui para a melhoria continuada da qualidade dos programas do SNPG, ao mesmo tempo que serve para prestar contas, de modo direto e transparente, à sociedade brasileira. ${ }^{5}$

Contudo, considerando-se o tamanho da população do país, o Brasil está longe de uma situação adequada e necessária para a capacitação de pessoal pós-graduado. Temos hoje cerca de oito doutores a cada 100 mil habitantes, e essa relação é insuficiente em face dos desafios nacionais. Atualmente, são 698 cientistas e engenheiros por milhão de habitantes. Para atender a esses desafios, o tamanho do sistema precisa ser ampliado significativamente, a fim de atingir os níveis dos países que compõem a OCDE. ${ }^{6}$

Em que pese os bons resultados obtidos pelo SNPG, o atual sistema avaliativo requer aperfeiçoamentos conceituais e operacionais. Transformações significativas nos cenários nacionais e internacionais requerem novas ações das comunidades acadêmica, científica, tecnológica e de inovação, sinalizando também para a necessidade de atualização dos procedimentos e dos critérios do modelo de avaliação.

Demandas de várias ordens emergem, como aquelas relacionadas à consolidação, à internacionalização, à inovação e à interação estruturada do SNPG com setores extra-acadêmicos, em especial àqueles diretamente conectados com o processo de transferência de conhecimento para a sociedade e maior protagonismo no processo de desenvolvimento socioeconômico, bem como na redução das assimetrias regionais.

A capacitação de pessoal por meio da pós-graduação tem um vasto conjunto de desdobramentos que precisa ser explicitado para maior clareza dos cenários atuais, nos seus vários contextos: inclusão social, ambiental e tecnológica e geração de renda. Nesses contextos, é preciso ter em conta as múltiplas dimensões da pós-graduação. Além disso, sua função social explicita-se por meio de um vasto conjunto de produtos e processos que chega difuso até a sociedade.

É inegável que a solidez do SNPG foi construída com importantes aportes de recursos da sociedade. É agora o momento de avançar na consolidação desse sistema, delineando ações que redundem na ampliação da formação de pessoal para atuar na qualificação do país como sociedade do conhecimento. ${ }^{7} \mathrm{O}$ processo de avaliação contribuiu para que fosse possível compatibilizar os cenários qualitativos internacionais e os anseios da sociedade brasileira. 
Nesse sentindo, a comissão do PNPG apresentou, em relatório aprovado pelo Conselho Superior da Capes em 2018, um estudo que ampliou os objetivos do SNPG. O desenvolvimento desse estudo teve como ponto de partida uma ampla consulta às associações e aos organismos representativos da ciência, tecnologia e inovação, em particular o Conselho Técnico-Científico, que resultou em uma série de definições sobre a pós-graduação como sociedade e comunidade científica, definindo com clareza seu novo foco e objetivo.

Ao longo das últimas décadas, o foco da pós-graduação do país foi, basicamente, a formação de docentes e pesquisadores para o próprio sistema acadêmico nacional. Diante dos novos cenários, o SNPG deve ampliar a sua atuação na formação de pessoal qualificado para todos os segmentos da sociedade, com foco no desenvolvimento econômico, social e ambiental.

O principal objetivo dos cursos de pós-graduação deve ser formar mestres e doutores capazes de enfrentar novos desafios científicos com independência intelectual, contribuindo para o progresso científico, tecnológico, econômico e social do Brasil como nação independente, imersa em um mundo globalizado em rápida evolução. A pós-graduação deve ser estabelecida em um ambiente que estimule o avanço e o desbravamento das fronteiras do conhecimento, sem imposição de barreiras disciplinares e com atenção à sociedade, promovendo o diálogo entre pares em nível nacional e internacional. $^{8}$

O processo de avaliação de programas stricto sensu no Brasil é realizado em dois momentos: na submissão de propostas de cursos novos (APCN) e na avaliação periódica quadrienal. As avaliações são realizadas por membros representantes da comunidade acadêmica para cada área de conhecimento. Nelas, são atribuídas notas aos programas em uma escala de 1 a 7 , reservando-se as notas 6 e 7 para os cursos de excelência e que tenham doutorado. A análise de mérito é feita a partir de diretrizes e normas estabelecidas pela Capes em consonância com a política nacional de pósgraduação.

Notas inferiores a 3 reprovam a entrada de novos programas no sistema, bem como desativam programas em funcionamento. Uma vez aprovado, todos os programas são considerados de qualidade; as notas 3, 4 e 5 apenas atestam a posição dos em relação à classificação dos demais programas dentro de sua área de avaliação. Notas 6 e 7 devem representar os programas que alcançam desempenho equivalente aos centros de referência internacional. Dessa forma, a premissa é a de que a nota do programa esteja atrelada ao grau de maturidade que o programa atingiu dentro do sistema.

$8 \quad$ Ibidem. 
A avaliação pauta-se pelo mesmo conjunto de quesitos e itens avaliativos, no intuito de promover a equivalência de desempenho entre programas com as mesmas notas em diferentes áreas de avaliação.

A ficha de avaliação é o instrumento utilizado pelas comissões avaliativas para a padronização dos quesitos. No quadriênio 2013-2016, a ficha apresentou os seguintes quesitos: "Proposta do programa"; "Corpo docente"; "Corpo discente"; "Teses e dissertações"; "Produção intelectual"; e "Inserção social". A cada item de avaliação são atribuídos conceitos: "muito bom”, "bom", "regular", "fraco" e "insuficiente".

A métrica e o peso atribuído a cada quesito variam conforme a área de avaliação. Para a atribuição de nota, os avaliadores baseiam-se no regulamento da avaliação, que estabelece, por exemplo, que os itens "Corpo discente" e "Produção intelectual" são determinantes para a definição dos limites da nota a ser atribuída.

O desempenho do programa é comparado ao desempenho médio dos demais programas daquela área de avaliação, já que, na maior parte das vezes, há uma divisão por faixas percentuais para a atribuição dos conceitos. A comparação entre programas da mesma área faz com que as métricas e os pesos atribuídos aos itens de avaliação tenham que se readequar ao longo dos anos, já que não é possível que todos os programas alcancem a mesma nota, ou seja, sempre terão programas com notas inferiores ocupando a última posição da escala de classificação.

Dessa forma, pode-se dizer que a avaliação realizada pela Capes é ao mesmo tempo criteriosa e normativa, uma vez que utiliza critérios iguais para todos. No entanto, estimula a competição e impõe uma dinâmica de alteração e elevação de padrões de qualidade. Ademais, é regulatória, pois uma avaliação insatisfatória resulta no descredenciamento do programa mal avaliado. ${ }^{9}$

3. Perspectivas da avaliação multidimensional da Capes e os desafios da área do Direito

Conforme verificado, a avaliação dos PPGs baseia-se em um modelo único, com uma escala nacional e com padrões e critérios que, em princípio, são uniformes, mas que sofrem pequenas adaptações para atender às especificidades das diferentes áreas. $\mathrm{O}$ modelo atual gera uma nota única, considerando que se trata de um sistema nacional de pós-graduação, que exige uma avaliação que parte de uma concepção de qualidade preestabelecida e que produz resultados consistentes e comparáveis. Esse modelo, muitas vezes operacionalizado de forma rígida, nem sempre considerava diferenças de contexto 
e, por consequência, limitava a diversidade da oferta e acentuava assimetrias geográficas e sociais existentes.

AComissão Especial de Acompanhamento do PNPG 2011-2020 apresentou em fevereiro de 2020 uma proposta de aprimoramento da avaliação da pós-graduação brasileira. A proposta é de um modelo multidimensional. Ao final do ciclo avaliativo, cada PPG terá um resultado para cada uma das 5 dimensões de avaliação, permitindo, segundo a Comissão, avaliar os respectivos e diferentes desempenhos em cada uma delas, reconhecendo, assim, a diversidade e a qualidade dos PPGs. As 5 dimensões de avaliação seriam: "Formação de pessoal"; "Pesquisa"; "Inovação e transferência de conhecimento"; "Impacto na sociedade"; e "Internacionalização". ${ }^{10}$

A Comissão formulou os conceitos dos indicadores para cada dimensão do modelo. Na definição desses indicadores de avaliação, foram considerados, segundo a Comissão, tanto aspectos qualitativos como quantitativos, de forma a permitir uma apreciação abrangente dos PPGs. Enquanto muitos dos indicadores usados para a avaliação das dimensões "Formação de pessoal" e "Pesquisa" - que constituem a base do processo avaliativo - já estão bem estabelecidos, os indicadores para as dimensões "Inovação e transferência de conhecimento", "Impacto na sociedade" e "Internacionalização" podem ser vistos como representantes das mudanças mais significativas introduzidas nesse novo modelo.

Com relação à dimensão "Formação de pessoal", os indicadores fundamentais já são consolidados no âmbito do sistema de avaliação da Capes. Buscando aprimorar o modelo de avaliação vigente, a Comissão destacou alguns aspectos básicos que devem ser considerados:

- A atratividade do curso/programa, ou seja, sua capacidade de atrair candidatos externos à instituição e sua região;

- Taxa de sucesso na formação de mestres e/ou doutores;

- Diversidade da oferta de oportunidades de formação, ou seja, a riqueza de oportunidades oferecidas aos alunos para além das disciplinas curriculares e da vinculação a um projeto de pesquisa - seminários, conferências etc.;

- Envolvimento de docentes e pesquisadores externos em atividades formativas;

- Grau de satisfação dos alunos;

- Integração dos egressos no mercado de trabalho e seu desempenho. ${ }^{11}$

10 BRASIL. PNPG - Plano Nacional de Pós-Graduação - Relatório Final 2019. Ministério da Educação/ Capes: Brasília, 2019.

11 Ibidem. 
Em relação à dimensão "Pesquisa", ela tem lugar central no modelo corrente de avaliação e, por isso, a Comissão destacou a importância de um equilíbrio entre os indicadores qualitativos e quantitativos no processo de avaliação. A sugestão é que os aspectos básicos da avaliação nessa dimensão sejam:

- Regularidade e participação dos docentes e estudantes na produção científica;

- Impacto da produção;

- Excelência e relevância internacional;

- Participação em redes de pesquisa nacionais e internacionais de excelência;

- Captação de recursos nacionais e internacionais. ${ }^{12}$

$\mathrm{Na}$ dimensão "Inovação e transferência de conhecimento", a Comissão apontou que a avaliação da inovação depende de indicadores que captem a interação dos programas de pós-graduação com outros agentes da sociedade, incluindo as entidades públicas, as empresas privadas e as organizações do terceiro setor. Sabidamente, os programas de pós-graduação precisam se aproximar das demandas da sociedade, sem prejuízo para a formação de recursos humanos pós-graduados e para a produção científica de qualidade. É necessário estimular, também pela via do processo avaliativo, a aproximação dos programas de pós-graduação inclusive das empresas. Para estimular essa aproximação com os setores produtivos, sejam eles industriais, de serviços ou de gestão pública em todos os níveis, a Comissão destaca que devem ser desenvolvidos indicadores que avaliem a capacidade de apoio que os programas de pós-graduação possam oferecer às empresas em seus processos de inovação. É necessário ainda partir de um conceito amplo de inovação, que inclua também os diversos aspectos da inovação social.

A aproximação de programas de pós-graduação das atividades dos setores produtivos não acadêmicos depende, sobretudo, da organização da pesquisa e da pósgraduação como expressão de políticas institucionais e das políticas nacionais e regionais. As ações institucionais são essenciais para que o conhecimento se aproxime desses sistemas. O processo avaliativo e a produção de indicadores, inclusive de impactos, devem, assim, estimular essas ações. Nesse sentido, a Comissão entende que os aspectos básicos da avaliação na dimensão "Inovação e transferência de conhecimento" são:

- Inovações sociais relevantes;

- Inovações culturais relevantes;

- Inovações, projetos, produtos ou processos que contribuam para a sustentabilidade ambiental;

- Produção de valor por meio da transferência de conhecimento;

$12 \quad$ Ibidem. 
- Geração de startups;

- Cooperação na pesquisa científica e tecnológica com empresas. ${ }^{13}$

Em relação à dimensão "Impacto na sociedade", a Comissão propõe a análise e a definição de indicadores que possam captar o papel dos egressos e das pesquisas desenvolvidas na atração de novos investimentos, na geração de emprego e renda (spin-offs acadêmicos e startups geradas) e no desenvolvimento nacional, contribuindo para a redução das assimetrias intra e inter-regionais.

Os aspectos de relevância social e regionalização precisam ter uma importância mais decisiva na avaliação, considerando que a excelência também está relacionada a perspectivas sociais e regionais necessárias ao desenvolvimento do país. Os aspectos regionais não são considerados no processo avaliativo de cursos novos. Sobretudo em áreas mais periféricas, os programas acabam sendo avaliados com a mesma medida de cursos já consolidados de instituições com larga experiência em pesquisa e pós-graduação, localizados em regiões com melhores índices econômicossociais e com amplo investimento em ciência e inovação. É importante considerar as ações e as atividades fomentadas pelas FAPs e outras agências locais numa perspectiva de questões regionais estratégicas. Sem deixar de lado a qualidade e a busca por excelência, a avaliação precisa encontrar critérios e mecanismos que possam avaliar aspectos regionais e estratégicos, levando em conta a atuação das FAPs em programas e projetos para o desenvolvimento da região.

Além da produção intelectual e da formação qualificada de profissionais, os impactos sociais, econômicos, ambientais e tecnológicos produzidos pelos PPGs devem adquirir peso significativo dentre os critérios de avaliação. Assim, a inserção social deve ser valorizada: pesquisa aplicada à sociedade que gere melhoria na qualidade de vida da população e impacto positivo em segmentos da sociedade, mercados ou organizações.

Desse modo, não somente os programas ligados às ciências humanas e sociais podem ser beneficiados, pois o produto a ser avaliado é a solução de um problema (ou gargalo) apresentado pela sociedade, o que geralmente é complexo e requer interdisciplinaridade para sua solução. Assim, seria incentivada não apenas a produção de um artigo (paper) qualificado - importante por acrescentar conteúdo original e inovador ao estado da arte -, mas, também, a solução de um problema indicado pela sociedade, solução essa que pode surgir como uma política pública, uma melhoria de processo ou de produto, um software, um projeto de lei, uma patente, e assim por diante. Dessa forma, além de estimular o senso crítico pela metodologia científica validada e reprodutível, desperta-se a responsabilidade social do discente. Além disso, aumenta-se

$13 \quad$ Ibidem. 
a sintonia social da universidade, do instituto ou do centro de pesquisa, popularizando a ciência e permitindo à sociedade perceber com mais clareza o retorno do investimento feito na educação superior, especialmente na pós-graduação. ${ }^{14}$

Com relação à dimensão "Internacionalização", no relatório final da Comissão do PNPG de 2018, foi ressaltada a necessidade de valorizar PPGs que investem na internacionalização por meio de projetos duradouros, abrangendo todos os envolvidos na pós-graduação, incluindo o corpo técnico e administrativo. Além do estabelecimento de relações institucionais com programas de pós-graduação de outros países, devem ser valorizados, segundo a Comissão, o fluxo in e out de discentes e pesquisadores, projetos de pesquisa conjuntos, dupla titulação de estudantes, oferta de disciplinas conjuntas e cursos em inglês ou em outras línguas.

A internacionalização não deve ser um fim em si mesma, mas um meio para o continuado enriquecimento dos programas e de seus atores, bem como para o estabelecimento de um número cada vez maior de projetos e colaborações internacionais. A internacionalização efetiva e enriquecedora de um programa deve ser representada por um conjunto de ações fruto da reflexão dos seus membros e que, juntas, devem servir, entre outros, para alargar as fronteiras das pesquisas daquele programa, expandir o conhecimento e a experiência profissional de seus estudantes e demais atores e aumentar a visibilidade da produção do programa. Nesse sentido, os seguintes aspectos devem ser considerados no processo de construção de indicadores:

- Financiamento internacional para projetos de pesquisa sob liderança do curso/programa ou em associação;

- Relevância da produção em cooperação internacional;

- Visibilidade do curso/programa em nível internacional conforme indicadores internacionais de desempenho;

- Atratividade em nível internacional de alunos, pós-docs e docentes/pesquisadores. ${ }^{15}$

Avaliar os impactos dessas ações de internacionalização deve resultar em formulação e definições para o aperfeiçoamento de políticas institucionais, considerando as perspectivas científica, econômica e cultural do país.

\section{Conclusão}

O sistema de avaliação passou por mudanças estruturais durante as últimas décadas e continua sendo aperfeiçoado, com os objetivos de certificar a qualidade da pós-graduação brasileira e identificar assimetrias regionais e de áreas estratégicas do

\footnotetext{
Ibidem.

15 Ibidem. 
conhecimento no SNPG, a fim de orientar ações de indução na criação e expansão de programas de pós-graduação no território nacional e na formulação de políticas para a área de pós-graduação, bem como para o direcionamento das ações de fomento.

No momento, o sistema de avaliação está sendo aperfeiçoado com uma proposta de modelo multidimensional, pois o modelo atual é, muitas vezes, operacionalizado de forma rígida, nem sempre considerando diferenças de contexto e das áreas e, por consequência, limitando a diversidade da oferta e acentuando assimetrias geográficas e sociais.

Acredita-se, portanto, que, com um resultado para cada uma das 5 dimensões da nova avaliação, será possível avaliar as os diferentes desempenhos em cada uma delas e, desse modo, reconhecer a diversidade e a qualidade dos PPGs.

\section{Referências}

BRASIL. Relatório Técnico DAV: Avaliação Multidimensional PPG 2019. Brasília: Ministério da Educação/Capes, 2019.

BRASIL. PNPG - Plano Nacional de Pós-Graduação: Relatório Final 2019. Brasília: Ministério da Educação/Capes, 2019.

BRASIL. Plano Nacional de Pós-Graduação: PNPG 2011-2020. Brasília: Ministério da Educação/ Capes, 2010. 